\title{
Status of the PALM-3000 high order adaptive optics instrument
}

\author{
Rick S. Burruss ${ }^{* a}$, Richard G. Dekany ${ }^{b}$, Jennifer E. Roberts ${ }^{a}$, J. Chris Shelton ${ }^{a}$, J. Kent Wallace ${ }^{a}$, \\ Jonathan A. Tesch ${ }^{\mathrm{a}}$, Dean L. Palmer ${ }^{\mathrm{a}}$, David Hale ${ }^{\mathrm{b}}$, Randall Bartos ${ }^{\mathrm{a}}$, Kevin M. Rykoski ${ }^{\mathrm{c}}$, \\ Carolyn M. Heffner ${ }^{\mathrm{c}}$, Jamey E. Eriksen ${ }^{\mathrm{c}}$, Fred Vescelus ${ }^{\mathrm{a}}$ \\ a Jet Propulsion Laboratory, California Institute of Technology, Pasadena, CA, USA 91109; \\ ${ }^{\mathrm{b}}$ Caltech Optical Observatories, California Institute of Technology, Pasadena, CA, USA 91125; \\ ${ }^{c}$ Palomar Observatory, California Institute of Technology, Palomar Mountain, CA, USA 92060;
}

\begin{abstract}
We report on the status of PALM-3000, the second generation adaptive optics instrument for the 5.1 meter Hale telescope at Palomar Observatory. PALM-3000 was released as a facility class instrument in October 2011, and has since been used on the Hale telescope a total of over 250 nights. In the past year, the PALM-3000 team introduced several instrument upgrades, including the release of the $32 \times 32$ pupil sampling mode which allows for correction on fainter guide stars, the upgrade of wavefront sensor relay optics, the diagnosis and repair of hardware problems, and the release of software improvements. We describe the performance of the PALM-3000 instrument as a result of these upgrades, and provide on-sky results. In the $32 \times 32$ pupil sampling mode $(15.8 \mathrm{~cm}$ per subaperture), we have achieved K-band strehl ratios as high as $11 \%$ on a $14.4 \mathrm{~m}_{\mathrm{v}}$ star, and in the $64 \times 64$ pupil sampling mode $(8.1 \mathrm{~cm}$ per subaperture), we have achieved K-band strehl ratios as high as $86 \%$ on stars brighter than $7^{\text {th }} \mathrm{m}_{\mathrm{v}}$.
\end{abstract}

Keywords: adaptive optics, Palomar, PALM-3000, P3K, wavefront sensing

\section{INTRODUCTION}

PALM-3000 is the second generation adaptive optics facility instrument for the 5.1 meter Hale telescope at Palomar Observatory ${ }^{[1]}$, specifically designed for near-infrared high-contrast imaging and spectroscopy of large companions and disk material near bright stars. The flexibility to observe using guide stars as faint as $\mathrm{m}_{\mathrm{v}} \sim 18$ was also designed into the instrument, but this functionality was delayed in an effort to get on sky quickly. PALM-3000 was commissioned in early 2011, and released as a facility class instrument to the general Palomar observing community in October 2011.

Working under an agreement between Palomar Observatory, Caltech Optical Observatories (COO), and the Jet Propulsion Laboratory (JPL), a small team of engineers lead by JPL was formed to work with the Palomar staff to maintain, upgrade, and operate PALM-3000. General maintenance, installation, and operation of PALM-3000 are provided entirely by the Palomar Observatory staff. JPL leads the effort to support continuing high-level maintenance, on-call operations support, new instrument interfaces, and instrument upgrades.

Six science instruments have been used with PALM-3000 to date, 2 of which are available to the general Palomar observing community (Pharo, Swift), 2 of which are private (P1640, Fiber Nuller), and 2 of which are still under development (Tmas, Stellar Double Coronagraph). To date PALM-3000 has been installed on the telescope 28 times, and has been the primary instrument for 242 nights. Science data obtained with PALM-3000 has been used in the publication of high-contrast imaging (Hinkley ${ }^{[2]}$, Wahl ${ }^{[3]}$ ) and of spectra of multiple exoplanets orbiting a single star $\left(\right.$ Oppenheimer $\left.{ }^{[4]}\right)$. While supporting the science activities, the PALM-3000 engineering team has also worked to improve the performance of the instrument in an effort to enhance the existing high contrast performance, as well as to provide the ability to correct the atmosphere on faint guide stars for projects such as Kepler follow-up and solar system science.

Upon release as a facility class instrument, PALM-3000 was underperforming on bright stars versus the expected error budget prediction ${ }^{[5]}$. Using strehl ratio from direct imaging as our performance metric, the average PALM-3000 K-band strehl ratio on bright stars was $75 \%$, with a best-case strehl ratio of $82 \%$, below the error budget prediction of $91 \%$ on

\footnotetext{
Adaptive Optics Systems IV, edited by Enrico Marchetti, Laird M. Close, Jean-Pierre Véran, Proc. of SPIE Vol. 9148, 914827 · @ 2014 SPIE CCC code: $0277-786 \mathrm{X} / 14 / \$ 18 \cdot$ doi: $10.1117 / 12.2055538$
} 
bright stars in median seeing. By the summer of 2012 several issues were uncovered that were determined to likely impact PALM-3000 performance. In addition, several upgrades were added to PALM-3000 to improve bright guide star performance, increase observing efficiency, and provide for fainter guide star science. We report on the status of these upgrades, and show the current PALM-3000 on-sky performance.

\section{PALM-3000 INSTRUMENT UPGRADES}

Many components have to work well together to achieve the highest possible performance in an instrument as complex as PALM-3000. The PALM-3000 engineering team identified and tested many of these components, and fixed several issues as they were discovered. The following three work efforts were significant in their impact on observing efficiencies and performance enhancements.

\subsection{Wavefront Sensor Relay Optics}

During PALM-3000 commissioning it was determined that the instrument's closed loop residual rms error was higher by 40-80 nanometers on blue stars $(\mathrm{O}, \mathrm{A})$ as opposed to red stars, leading to a drop of $\sim 10 \%$ in strehl ratio of the star's point spread function (PSF), due purely to a focus error. Tests in the lab confirmed the theory that source color was the cause. To allow for continued operations a work-around was developed to translate the entire wavefront sensor camera assembly along the focus direction, to null the incorrect focus term. The Palomar operators implemented this workaround on blue stars starting in September 2012, while the JPL team began to determine where the error was happening, and what was needed to solve it.

As part of the original PALM-3000 Shack-Hartmann design, a standard pair of lenses were used downstream of the lenslets to reimage the lenslet spots onto the wavefront sensor CCD in the desirable spacing and centration. Care was taken during the design to ensure the final magnification was correct and did not depend on the chosen set of lenslets or the characteristics of the source. A more careful inspection of the lateral color term in our Zemax model of the two relay lenses showed a color dependent distortion that we had not previously seen. This distortion caused a slightly larger magnification on bluer sources, which caused the lenslet spots to appear to spread out further than designed at the outer edge of the pupil. The Shack-Hartmann wavefront sensor measured this effect as focus, causing an incorrect focus term to be applied during closed loop correction.

Once the source of the error was determined, we agreed to fix the problem under the following constraints: the new relay must fit in the same space and maintain the existing demagnification ratio, it must minimize distortion as a function of wavelength, and it must be easy to align. We chose to make the new relay tele-centric, in an effort to make the new relay easier to align and test. We settled on a solution that incorporated a custom doublet from Optimax Systems Inc. and an off-the-shelf triplet lens from Edmund Optics. A custom opto-mechanical baseplate, mounting platform, and baffle was designed at JPL to hold the two optics. The mounting platform was designed to easily modify the locations of each optic as well as the entire platform with respect to the underlying focus stage.
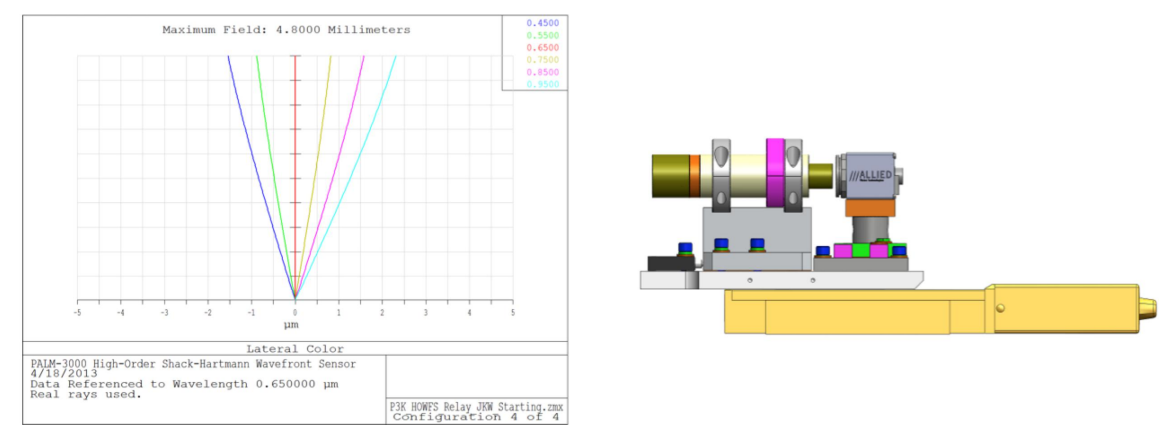

Figure 1. Wavefront Sensor Relay: left. The lateral color error as measured by Zemax of the original wavefront sensor relay design. The lateral color error term over the span of $450 \mathrm{~nm}$ to $950 \mathrm{~nm}$ is only $+/-2 \mathrm{um}$, but the resulting magnification change in the Shack-Hartmann camera over this range induces a significant focus error term. right. The final design of the new relay showing the mounting hardware, the baffle tube, the alignment camera, and the wavefront sensor focus stage. 
With the new relay installed and verified in the lab, we tested the closed loop operation on stars from red (M) to blue $(\mathrm{O})$, and we found that the focus error was completely solved by the new relay. The Palomar operators were then able to stop using the work-around solution, increasing PALM-3000 observing efficiency. And since the target star color is rarely known exactly, we suspect that strehl ratios have improved for all stars bluer than $\mathrm{K}$ with the new optics in place.

\subsection{Wavefront Sensor Camera}

The PALM-3000 wavefront sensor detector is an E2V CCD-50 with 128x128 pixels, shuttered using a split-frame transfer and read out through 16 amplifier channels. The detector is contained in a Little Joe camera unit manufactured by SciMeasure Analytical Systems Inc. The controller, also manufactured by SciMeasure, is a $3 \mathrm{U}$ chassis housed in one of four PALM-3000 racks connected via 30 foot cables to the optical bench. The controller generates the output clock signals and biases, while accepting the 16 video channel inputs from the camera head. Both output and input signals travel over cables that were initially 30 feet in length.

The CCD was originally tuned to optimize low light level performance at the slowest CCD frame rates. High frame rate performance, necessary for bright guide stars, was good, but we found that on sky, performance suffered due to higher noise along the channel seams in the raw pixel data. Working with SciMeasure, we formulated a plan to test and tune the highest frame rates of the $\mathrm{CCD}(2 \mathrm{KHz})$ while not degrading performance in the lower frame rates $(30 \mathrm{~Hz})$.

During testing we verified that the channel seams in the raw pixel data were indeed noisier than the rest of the detector area. The first 2-3 columns of each channel suffered a non-linear response in the presence of flux. In addition, we also determined that the CCD suffered from two additional problems: a background shift on each channel in the presence of flux (DC restoration error), and a smear of flux in the fast read out direction (charge transfer efficiency error).
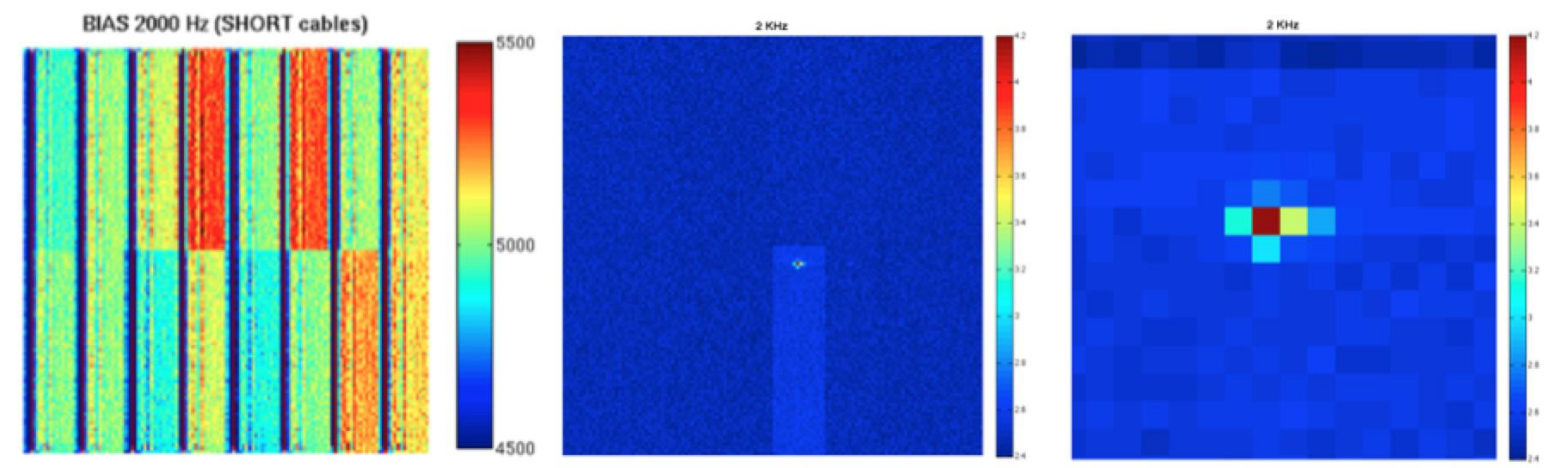

Figure 2: Wavefront sensor CCD problems at 2 KHz: left: bias frame showing strong reduction in signal at each of the 16 channel seams. In the presence of flux, these seams are non linear in response. When observing on sky, the seams are the first areas to go unstable in closed loop operation when running faster than $1250 \mathrm{~Hz}$ in low light conditions. center: A single spot focused on one of the CCD channels shows an increase of the background in this channel only. This is classic DC restoration error. right: Zoomed in on the single focused spot shows smear in the horizontal fast read out direction. These effects were all caused by the extra long cables inducing reflections, crosstalk, and timing errors, and increasing the effective clock driver impedance.

We determined that these errors could be minimized by reducing the length of the output driver cables and the input video cables to 18 feet (from the original 30 feet), and tuning the clock driver to optimize the clock signals to the new cable lengths. We also determined that the clock driver cable was in need of an upgrade to replace individual wires with coax and match the impedance between the driver source, the coax wire, and the RC filters. The video cable was also in need of work to match source and coax impedance. This cable work was completed in parallel to our CCD testing activities.

We took the new cables to Palomar and worked on the circuits of the CCD camera head and controller with the instrument in the adaptive optics lab. In addition to optimizing the CCD electronics to the new cables, we also removed a source of heat from the camera head, which reduced the dark current from $780 \mathrm{e}^{-} / \mathrm{second}$ to $80 \mathrm{e}^{-/} / \mathrm{second}$. Once the work on the CCD electronics was completed, and the values for CCD clamp and sample were optimized, we found a significant improvement in the column seam behavior. The DC restoration and horizontal smear errors are solved. The 
dynamic range of the CCD is slightly improved, and the non-linear response of the channel seams is slightly better, though not yet completely clean. Our hope is that these enhancements will lead to an improvement in overall PALM3000 performance. In particular we are hopeful that our average K-band strehl ratio of $82 \%$ will improve as a result of this work. Unfortunately the wind conditions and seeing were very poor during our one engineering night to test this upgrade, so we have not yet been able to categorize the CCD upgrade work.
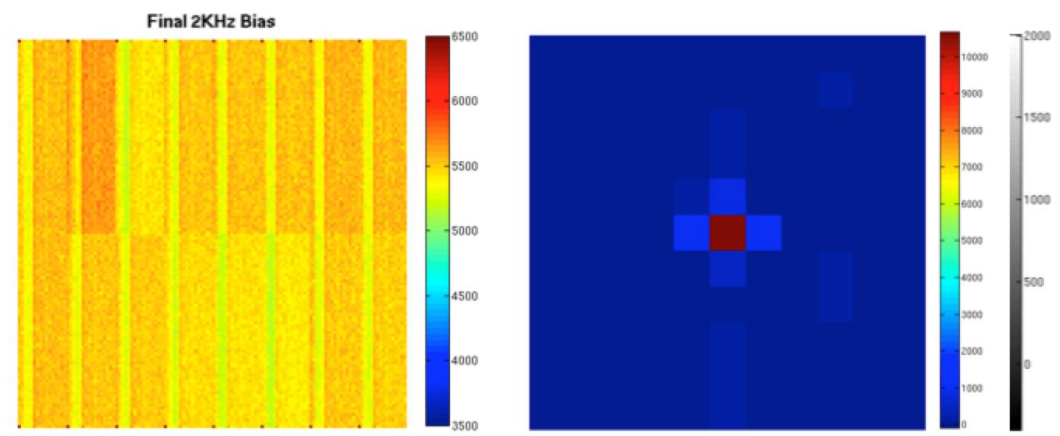

Figure 3: Wavefront sensor CCD upgrade: left: $2 \mathrm{KHz}$ bias frame showing the 16 channels and seams after the upgrade work. The seam artifacts are dramatically improved, and all reflections from the seams themselves are eliminated. right: The smear of flux in the horizontal fast read direction is fixed, and the DC restoration is significantly improved.

\subsection{Software Upgrades}

The PALM-3000 engineering team has dedicated nearly half of each year to software upgrades, and these fall into two categories: operations control (which lead to observing efficiencies) and real-time control (which lead to performance improvements). Operations control software includes all the automations needed to setup the instrument on a guide star after a telescope slew, and then maintain the instrument on the guide star once the target is locked.

The Palomar Observatory support astronomers are charged to startup and align the instrument during the day, and help the operators and scientists during the first few hours of observing. The Palomar night assistants are the operators, and are charged to run the instrument for the science teams. Great effort was put into the PALM-3000 automation and graphical user interface (GUI) designs to make their jobs as easy as possible due to the complexity of the PALM-3000 instrument with its two adaptive mirrors in a dual mirror real-time control algorithm, 3 different pupil sampling observing modes, and 15 motors controlling critical optics in various locations.

The PALM-3000 engineering team has steadily worked to fix software bugs, improve existing software functionality, and add new functionality. Since the delivery of the instrument in 2011, 12 significant updates to the control software have been released. In addition to numerous bug fixes that have increased operations efficiency, new automations have been released to help streamline the target acquisition process. These new automations include; the auto registration of the deformable mirror to the wavefront sensor in all pupil sampling modes, the auto centering of the optical beam in the wavefront sensor with respect to the spatial filter, and the auto optimizing of the system when changing wavefront sensor camera rates. Both the bug fixes and the new automations have lead to a notable reduction in target acquisition times.

The ability to use variable guide star brightness is provided by a user-selectable pupil sampling mode designed into the Shack-Hartmann wavefront senor. To achieve the best possible bright star performance and highest contrast, PALM3000 was first delivered to Palomar Observatory with the finest pupil sampling scale of 64 subapertures across the pupil (64x). The hardware, optics, and automation software for the fainter observing modes were all in place when the instrument was first delivered to Palomar, but the real-time control software was purposely left unfinished.

To observe on guide stars in the $8-12 \mathrm{~m}_{\mathrm{v}}$ range, the 32 subapertures per pupil sampling mode $(32 \mathrm{x})$ was delivered in the spring of 2013. Upgrades and bug fixes to both the operations and the real-time software were implemented to enhance this observing mode since its release with the performance gains for each upgrade noted in Figure 4 . To observe on stars in the $12-18 \mathrm{~m}_{\mathrm{v}}$ range, the 8 subapertures per pupil sampling mode $(8 \mathrm{x})$ was delivered in the spring of 2014 . 
We also continue to update our innovative reconstructor pipeline software ${ }^{[1]}$ when new pupil sampling modes are delivered, and as more experience with these modes are acquired. This software serves to optimize the closed loop control based on the pupil illumination pattern and other observing parameters. A Bayesian implementation was released in the spring of 2013 to better optimize the closed loop performance on faint stars or poor seeing, and the effect this has on PALM-3000 performance is seen in our on-sky results (Figure 4).

\section{CURRENT PERFORMANCE}

The performance of PALM-3000 as measured using strehl ratio has steadily improved, in both the $64 \mathrm{x}$ and $32 \mathrm{x}$ observing modes, since the instrument was delivered in 2011. In addition, the observing efficiency has increased, and the system downtime due to software or hardware failures has remained below our goal of $5 \%$ per observing semester.

\subsection{On Sky Results}

PALM-3000 K-band strehl ratios are shown in Figure 4, with the error budget predictions for the 64x, 32x, and 8x pupil sampling modes. As of June 2014, our average K-band strehl ratio in median conditions at zenith is $82 \%$ (152 nm rms), with a best-case PSF of $86 \%(132 \mathrm{~nm} \mathrm{rms})$. We're hopeful that the work put into the upgrade of the wavefront sensor $\mathrm{CCD}$ will serve to boost these results even higher.

Performance in the 32x pupil sampling mode on guide stars in the $7-9 \mathrm{~m}_{\mathrm{v}}$ range is below expectations. However, a recent bug fix in software that controls this mode may solve this problem. The advantage of using our Bayesian implementation of the reconstructor pipeline code is clearly evident in the $32 \mathrm{x}$ pupil mode on stars fainter than $9 \mathrm{~m}_{\mathrm{v}}$ in the data acquired in June and September 2013.

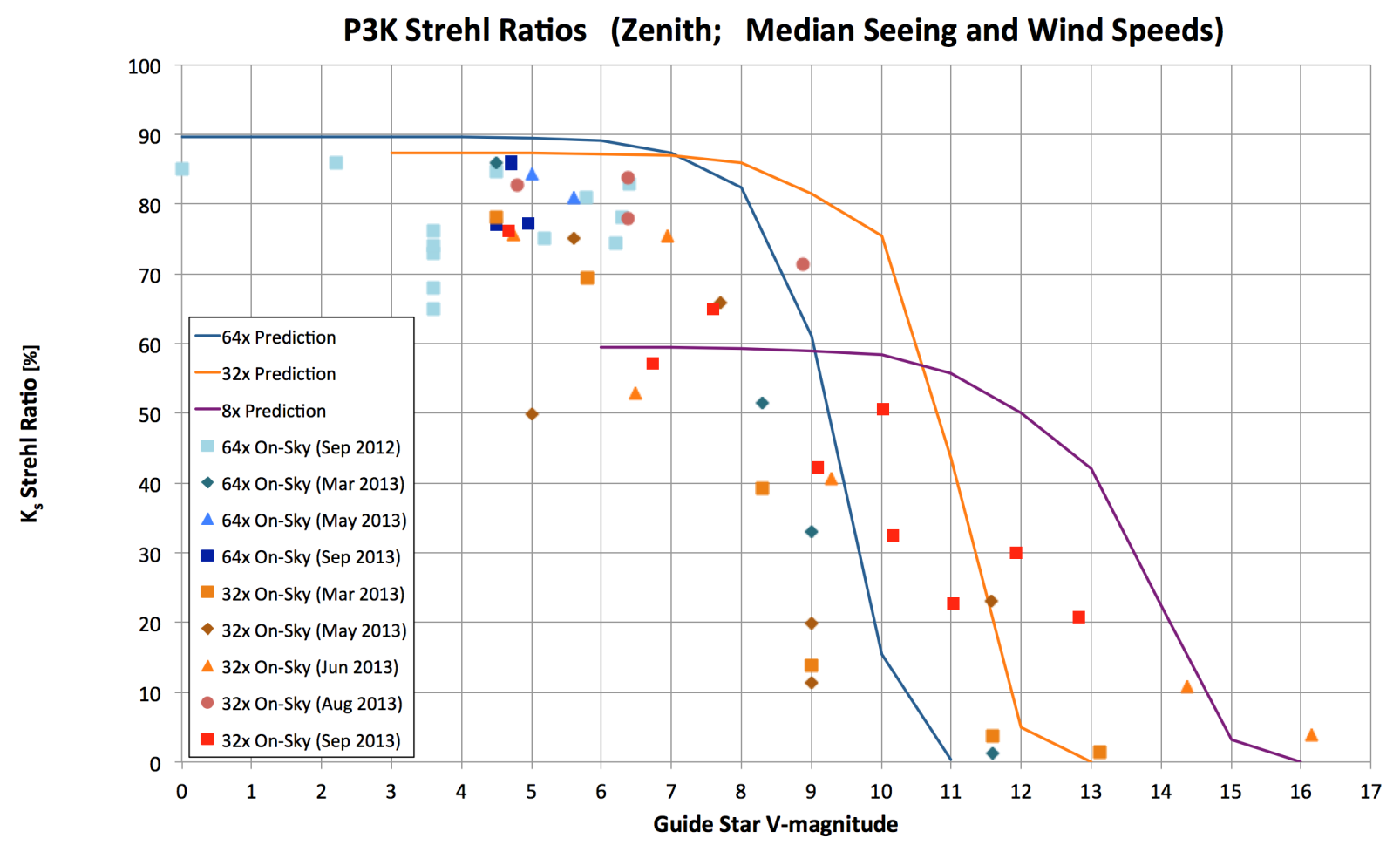

Figure 4: PALM-3000 performance chart: $64 \mathrm{x}$ and $32 \mathrm{x}$ Ks-band strehl ratio as a function of guide star brightness. Only data points taken in median or better seeing and at zenith are shown. The error budget predictions for $64 \mathrm{x}, 32 \mathrm{x}$, and $8 \mathrm{x}$ are also shown. The data points taken on stars fainter than $12 \mathrm{~m}_{\mathrm{v}}$ exceed performance expectation because of our Bayesian implementation in the reconstructor pipeline software. The data points show the progression of PALM-3000 performance from late 2012 to late 2013 . All data taken after September 2013, over a total of 3 engineering nights, are unusable due to poor seeing and wind conditions. 

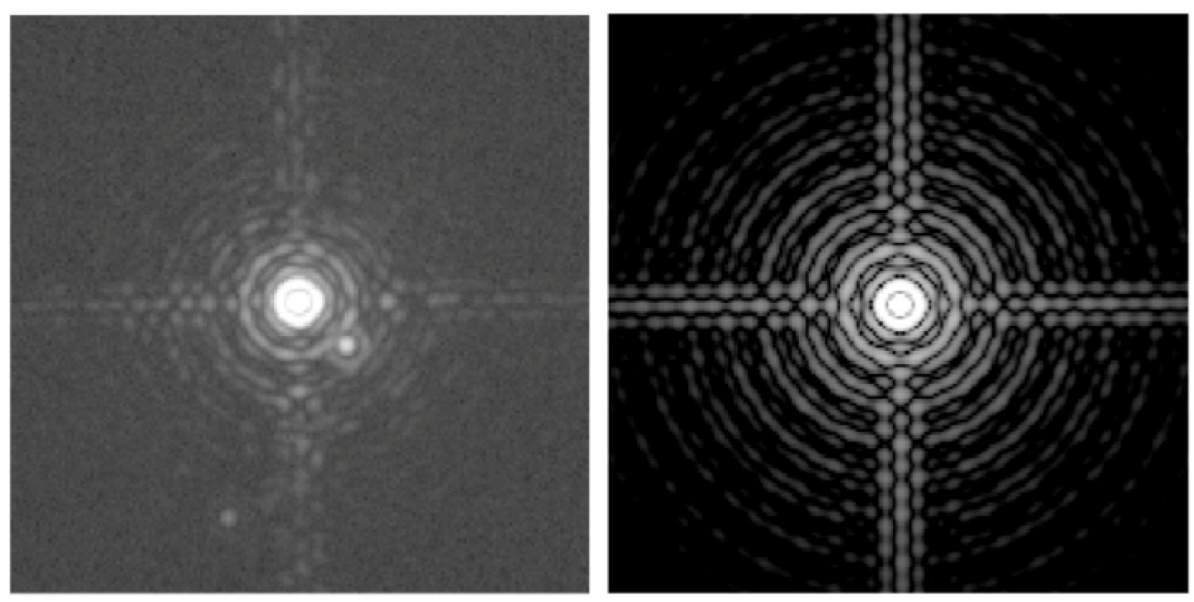

Figure 5: SAO 059858: left: $4.5 \mathrm{~m}_{\mathrm{v}} \mathrm{K} 0$ star near zenith on March 23, 2013 in BrGamma using Pharo imaging. Seeing (visible) = 0.81 ", 85 seconds total integration time, wavefront sensor camera rate $=2 \mathrm{KHz}$, strehl ratio $=86.01 \%$. This is our best strehl ratio to date. right: The perfect Pharo BrGamma PSF with the same pupil and imaging parameters.

The 8 subaperture per pupil observing mode (8x) was delivered in the spring of 2014 . We've had one night on the telescope so far to test this mode, but unfortunately the seeing and wind speeds were extraordinarily high on this night, so we were unable to obtain strehl ratio on any stars fainter than $8 \mathrm{~m}_{\mathrm{v}}$. However, we were able to close both our tip-tilt and deformable mirror loops on every star we tried, from 8 to $17.93 \mathrm{~m}_{\mathrm{v}}$. We are looking forward to our summer 2014 engineering time on the telescope to verify all 3 modes of pupil operation, and hopefully obtain our first strehl ratios in the $8 x$ pupil sampling mode.
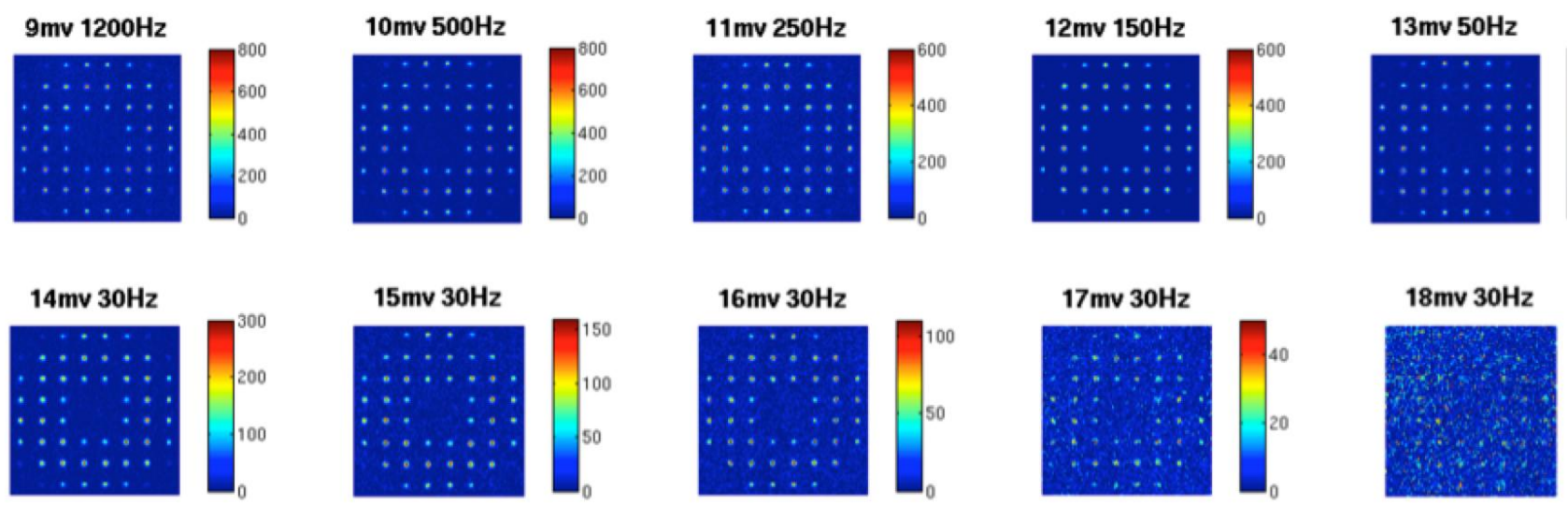

Figure 6: 8x pupil sampling mode: PALM-3000 8x Shack-Hartmann pixel data on guide stars from $9-18 \mathrm{~m}_{\mathrm{v}}$. Both the tip-tilt and deformable mirror loops were stably closed in all 10 cases, but the wind speeds and seeing were exceptionally poor on this engineering night, so we were unable to gather strehl ratio data. We are encouraged that both the tip-tilt and higher order correction were stable on an $18 \mathrm{~m}_{\mathrm{v}}$ target in such bad conditions.

\subsection{Operations}

The work to enhance operations control software, and the training achieved with the Palomar night staff, have resulted in a significant improvement in PALM-3000 observing efficiency. In October 2011, the typical setup time from the end of a telescope slew to the start of science observations in the 64x pupil sampling mode was 15-20 minutes per target. As a result of our upgrade work, and the tireless efforts of the Palomar Observatory night staff, the average setup time from 
the end of a telescope slew to the start of science observations is at most 10 minutes per target in $64 \mathrm{x}$, and typically closer to 5 minutes.

Target acquisition times are longer if the pupil sampling mode is changed, which can start during the telescope slew to minimize the delay. We are working on streamlining the operations control software to improve the acquisition process.

We have also worked to improve the reliability of various hardware components, including the high-order deformable mirror electronics, acquisition camera electronics, and various other hardware components and driver software. As a result, the total accumulated instrument downtime measured since PALM-3000 was delivered in 2011 is 3.8\% of the total available sky time. The original downtime requirement imposed on PALM-3000 was 10\%, with a goal of 5\%.

PALM-3000 Downtime

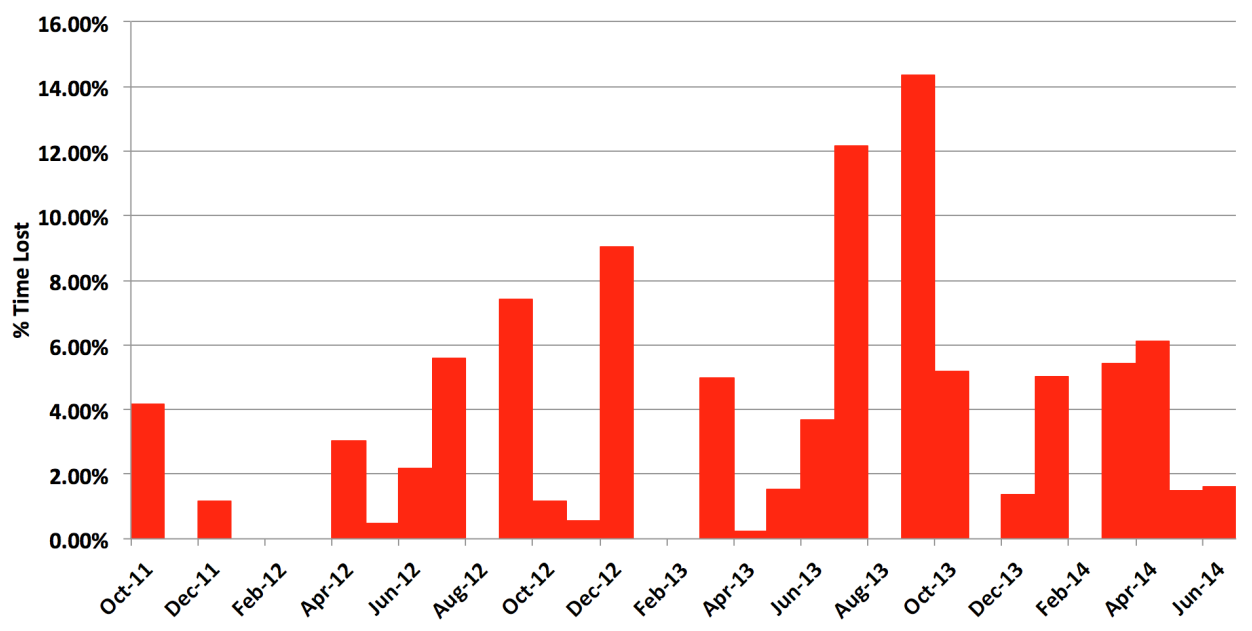

Figure 7: PALM-3000 downtime: The percentage of PALM-3000 downtime since it became a facility class instrument in October 2011. The average downtime over all 242 nights on sky is $3.8 \%$. The spike in the fall of 2013 is due to the failure of the high-order deformable mirror control electronics. We have since purchased spares of the failed components.

\section{PLANNED WORK}

The PALM-3000 engineering team has 3 more on-sky engineering nights on the Hale telescope in 2014. We are hopeful that we will find improved strehl ratios in all 3 pupil observing modes as a result of the work efforts described in this paper. We are planning more upgrades to the operations control software to improve the ease of switching between the three observing modes, in preparation for the upcoming full release of the $8 \mathrm{x}$ pupil sampling mode to the Palomar staff. The 5-10 minute target acquisition times currently enjoyed by the PALM-3000 users will be potentially increased by the release of the $8 \mathrm{x}$ observing mode.

Upgrades are also planned to the GUI software to help streamline the operator's environment, including new system diagnostic tools to guide the optimization of the PALM-3000 real-time control loop parameters. We plan to install new database hardware and software in 2015 to enhance the data recording process. Improved data recording will allow better debugging of problems during high-speed operations. And we plan to continue CCD testing and upgrade work to further enhance the fastest frame rate performance.

Finally, we are looking forward to the integration and first results of the Stellar Double Coronagraph, a novel new dual vector vortex coronagraph addition to the Pharo science camera. First light for this instrument was obtained in June 2014, and upgrades in the lab are ongoing. 


\section{CONCLUSIONS}

PALM-3000 is a state-of-the-art adaptive optics instrument for the Hale telescope at Palomar Observatory. It is operated entirely by the Palomar Observatory staff. In median or better seeing near zenith, the average K-band strehl ratio on stars brighter than $7 \mathrm{~m}_{\mathrm{v}}$ is $82 \%(151 \mathrm{~nm} \mathrm{rms})$. PALM-3000 performance is underperforming slightly in the $7-10 \mathrm{~m}_{\mathrm{v}}$ range in 32x pupil mode, and our team is working to resolve this issue. Using the 32x pupil sampling mode, PALM3000 exceeds performance expectations on stars from $10-13 \mathrm{~m}_{\mathrm{v}}$. The $8 \mathrm{x}$ pupil sampling mode, delivered in spring 2014, will allow correction on stars as faint as $18 \mathrm{~m}_{\mathrm{v}}$. The PALM-3000 engineering team will be working to categorize $8 \mathrm{x}$ performance in the summer of 2014. As we continue to improve the functionality, performance, and observing efficiency of PALM-3000, Palomar Observatory will continue to be regarded as offering one of the world's leading astronomical adaptive optics instruments, and PALM-3000 will remain a crucial part of the Hale telescope instrumentation suite.

\section{ACKNOWLEDGEMENTS}

This work was carried out at the Jet Propulsion Laboratory, California Institute of Technology, under contract with the National Aeronautics and Space Administration, and is based on observations obtained at the Hale Telescope, Palomar Observatory, as part of a continuing collaboration between the California Institute of Technology and NASA/JPL. We thank the staff of the Palomar Observatory and the Palomar Adaptive Optics team for their continued support.

Reference herein to any specific commercial product, process, or service by trade name, trademark, manufacturer, or otherwise, does not constitute or imply its endorsement by the United States Government or the Jet Propulsion Laboratory, California Institute of Technology.

We would like to thank the Palomar Observatory superintendent Dan McKenna for his generous support of PALM-3000. In particular we would like to thank the Palomar day crew members; Steve Kunsman, Greg Van Idsinga, Mike Doyle, and John Baker, for their tireless efforts installing the complex PALM-3000 instrument on the telescope (28 times so far), and then back to the lab again (28 more!). We would like to thank John Henning for his expert help with the Palomar Observatory software and hardware interfaces to PALM-3000. We would finally like to thank the excellent

night crew responsible for operating the most complex instrument ever run by Palomar staff: Jean Mueller, Kajsa Peffer, and Paul Nied. Thank you all for your generous, and continuing, support.

Finally we wish to extend our heartfelt appreciation and best wishes to Jean Mueller, who will be moving on to the next chapter in her life. Your patience, dedication, and expert eye has helped guide both PalAO and PALM-3000 at Palomar to the forefront of adaptive optics science.

\section{REFERENCES}

[1] Dekany, R., et. al., "Palm-3000: exoplanet adaptive optics for the 5-meter Hale telescope", The Astrophysical Journal, Vol. 776, 130D, 2013

[2] Hinkley, S., et. al., "The $\kappa$ Andromedae System: New Constraints On The Companion Mass, System Age \& Further Multiplicity", The Astrophysical Journal, Vol. 779, 153H, 2013

[3] Wahl, M., et. al., "Debris Disk Science with the Palomar ExAO System: First Results", IAU Proceedings, Symposium 299, Vol. 8, pp 72-73, 2013

[4] Oppenheimer, B., et. al., "Reconnaissance of the HR 8799 Exosolar System. I. Near-infrared Spectroscopy", The Astrophysical Journal, Vol. 768, 24O, 2013

[5] Roberts, J., et. al., "Results from the PALM-3000 high-order adaptive optics system”, SPIE, 8447, 2012 